\title{
Are Strokes Mostly Genetically Based or Acquired? Stenosis vs Hypoplasia of Vertebral Artery Anomalous as The Leading Cause of Stroke (Anatomical Study of VA Anomalies in Neck Three- Dimensional Computed Tomography Angiography)
}

Farzaneh Mohammadi ( $\nabla$ farzaneh.mohammadi@easternhealth.org.au )

Eastern Health

Hussein Soleimantabar

Shahid Beheshti University of Medical Sciences

Fatemeh Mohamadi

Emam Jafar Sadegh Hospital, Shahid Beheshti University of Medical Sciences

Sofia Sabouri

Shahid Beheshti University of Medical Sciences

\section{Research Article}

Keywords: Vertebral Artery, Anomalies, CT angiography, Significant Stenosis, Hypoplasia

Posted Date: August 23rd, 2021

DOI: https://doi.org/10.21203/rs.3.rs-791684/v1

License: (9) This work is licensed under a Creative Commons Attribution 4.0 International License. Read Full License 


\section{Abstract \\ Background}

A thorough understanding of the vertebral artery (VA) variations and course of the artery and its related branches could significantly enhance the efficacy and safety of interventions involving the neck and spine. Here, we aimed to investigate the incidence of various VA variations in individuals presenting with signs and symptoms of stroke with our neurology team. In this cross-sectional study, all patients referred to our specialized medical imaging center and underwent neck CT-angiography (CTA) were enrolled in this study for two years from September 2019 onward. Patients' information forms were obtained to collect demographic data.

\section{Results}

An experienced board-certified radiologist evaluated the CTA images, recording the characteristics of VA, regarding dominance and any possible anomalies. These included anomalous origin, anomalous route, anomalous branching, stenosis, hypoplasia, atresia, thrombosis, and dissection. A total of 125 patients (53.6\% females; mean age, $60.7(11-87)$ ) were included. In the case of VA dominance, $66.4 \%$ of cases were co-dominant while $16.0 \%$ were right, and $12.8 \%$ were left dominant. Overall, males had more VA anomalies than females, $24.8 \%$ and $17.6 \%$, respectively (in general, $42.4 \%$ of all participants) $(p=0.020)$. Among patients, $4.0 \%(n=5)$ had an anomalous origin and anomalous branching was reported in 5 patients (4.0\%). Among the variations, significant stenosis (18.4\%) and hypoplasia (17.6\%) were the most prevalent anomalies with the earliest one having the higher rate. While anomalous branching was reported in 5 patients $(4.0 \%)$.

\section{Conclusions}

VA variations are relatively common and should be addressed during procedures involving the neck region and would allow surgeons to avoid life threatening injury.

\section{Background}

The vertebral artery (VA) arises from the respective subclavian artery and connects the opposite site branch to form the basilar artery [1]. The VA is anatomically divided into four segments: V1, preforaminal; V2, foraminal; V3, atlantic; and V4 intracranial [2]. Anatomical variations mainly involve the distal segments of VA (V3 and V4). Several embryological explanations for these variations have been proposed in the literature [3].

With the growing use of vascular angiography, uncommon cases of hypoplasia, origin stenosis, and altered origin have thoroughly been diagnosed [4]. In Kauffman's study, it was reported that patients with 
VA stenotic origin are correlated with less risk of stroke through intraarterial angiography [5]. The VA origin and aortic arch branching patterns should be assessed in cases of cerebrovascular accidents and VA dissection [6].

In Kim's study, Vertebral Artery Hypoplasia (VAH) greatly contributes to a rising risk of Posterior cerebellar stroke (PCS) [7]. However, the rates of VAH reported in the literature vary markedly because there is currently no standard definition for this disorder [8]. The VA variations are highly significant from various clinical points of view. First of all, these variations could predispose some people to disorders impairing the blood supply to the head and neck. These could accompany other life-threatening vascular anomalies. In addition, spine surgeons and interventional radiologists might encounter problems in the VA during the procedure. It is noted that the incidence rate of VA injury in spine surgery has been reported to range from $0.3-8.2 \%[9-11]$.

Having a comprehensive understanding of the VA variations and course of the artery and its related branches preoperatively could significantly enhance the efficacy and safety of interventions involving the neck and spine, and subsequently enable surgeons to avoid injury [12]. In this study, we sought to investigate the incidence of various VA variations which would be helpful in head and neck surgery. Meanwhile, understanding the incidence of VA anomalies and variations and identifying which anomalies are more common in each gender in patients who present with signs and symptoms of stroke increases the early diagnosis and prevents life-threatening complications. We are assessing individuals who presented with signs and symptoms of stroke to our neurology team and referred to our imaging centre to investigate the possibility of stroke.

\section{Ethics approval}

Ethical approval for this Study and use of patients three-dimensional computed tomography angiography (CTA) images for collection and study was obtained originally from the local ethics committee in Shahid Beheshti university of medical sciences, Tehran, Iran. The Iran National Committee for Ethics in Biomedical Research approved continuing this study.

We conducted this study using data and CTA images from Tooska Medical Imaging center. All study participants provided written informed consent for studying their images. Patients explained regarding how their clinical data would be used in the study process. The patients' information remained confidential and identities were anonymous. All materials and methods were performed in accordance with the relevant guidelines and regulations and written informed consent for publication was obtained from patients.

\section{Materials And Methods \\ 2.1. Study design and participants}


The present study was a single-center retrospective observational investigation. Inclusion criterion for this study was: individuals who visited our neurology team with signs and symptoms of stroke or headache and dizziness in suspicious of posterior cerebellar stroke (PCS) were therefore referred to our specialized medical imaging centre (Tooska Medical Imaging Centre, Tehran, Iran). Exclusion criteria were patients in whom VA was not clear or patients without proper contrast injection. These patients underwent computed tomography (CT) angiography in September 2019, using the "census" sampling method. Eventually, 125 cases were applicable to this study after excluding subjects with undetectable VAs on their CTA. Patients' information forms were obtained to collect demographic details. Written consent forms were also obtained from each patient regarding how their clinical data would be used in the study process. The patients' information remained confidential and identities were anonymous.

\subsection{Image acquisition and interpretation}

A Lightspeed QXI four-slice CT scanner (GE, Milwaukee, WI, USA) was used in all examinations with a collimation of $1.25 \mathrm{~mm}$, pitch of 0.75 , and a table speed of $3.75 \mathrm{~cm}$. Visipaque 320 (iodixanol, GE Healthcare) was injected as the contrast agent with an infusion speed of 1-3 $\mathrm{ml}$ per second. An experienced board-certified radiologist evaluated all obtained CTA images, recording VA characteristics regarding dominance and any possible anomalies, including anomalous origin, anomalous route, anomalous branching, stenosis, hypoplasia, atresia, thrombosis, and dissection.

\subsection{Statistical analysis}

All raw data were analysed using SPSS version 24 (IBM Corporation; formerly SPSS Inc.), and nominal data were reported in terms of frequency and percentage with $95 \%$ confidence interval $(\mathrm{Cl})$. Numeric data were reported as mean \pm standard deviation (SD).

\section{Results}

In this section, we assess the prevalence of VA anomalies according to data from CT Angiography of patients who referred to the neurology team with signs and symptoms of PICA stroke. Initially, we studied the frequency of each variation followed by assessing the correlations between each anomaly. In the final analysis, 125 patients ( $53.6 \%$ females, $n=67$ ) were included after applying the inclusion and exclusion criteria with a mean age of 60.71 (range $25-87$ years). Patients were categorized into five groups based on age: under $30,31-40,41-60,61-80$, and over 80 years.

\section{1-Dominance}

In patients who presented the most frequent scenario was co-dominant with 83 cases followed by right dominant with 20 cases, the remaining ones being left dominant amounting to 16 cases. Hence, dominance was not identified in 6 patients (Table 1). 
Table 1

Dominancy in VA

\begin{tabular}{|llllll|}
\hline Age group Sex & Co-Dominant & Right Dominant & $\begin{array}{l}\text { Left } \\
\text { Dominant }\end{array}$ & Non-Dominant & Total \\
\hline$<30$ Male & 2 & 0 & 0 & 0 & 2 \\
Female & 0 & 0 & 0 & 0 & 0 \\
\hline $30-40$ Male & 5 & 0 & 0 & 0 & 5 \\
Female & 5 & 2 & 1 & 1 & 9 \\
\hline $40-60$ Male & 8 & 3 & 1 & 3 & 15 \\
Female & 18 & 4 & 2 & 0 & 24 \\
\hline $60-80$ Male & 17 & 6 & 8 & 1 & 32 \\
Female & 25 & 3 & 3 & 0 & 31 \\
\hline$>80$ Male & 1 & 1 & 1 & 1 & 4 \\
Female & 2 & 1 & 0 & 0 & 3 \\
\hline
\end{tabular}

3.2- Anomalous

\subsubsection{Anomalous origin}

In our study, only 5 cases with anomalous origin were found. 4 cases were Left VA originating from the aortic arch and in 1 case Right VA originating from Right Common Carotid Artery (Table 2). 
Table 2

Anomalous Origin of VA

\begin{tabular}{|lllll|}
\hline Age group Sex & No Anomalous & Left VA Originating from Aortic Arch & Others & Total \\
\hline$<30$ Male & 1 & 1 & 0 & 2 \\
Female & 0 & 0 & 0 & 0 \\
\hline $30-40$ Male & 5 & 0 & 0 & 5 \\
Female & 9 & 0 & 0 & 9 \\
\hline $40-60$ Male & 15 & 0 & 0 & 15 \\
Female & 21 & 2 & 1 & 24 \\
\hline $60-80$ Male & 31 & 1 & 0 & 32 \\
Female & 31 & 0 & 0 & 31 \\
\hline$>80$ Male & 4 & 0 & 0 & 4 \\
Female & 3 & 0 & 0 & 3 \\
\hline
\end{tabular}

\subsubsection{Anomalous route}

In this study, only one case was reported as both VA entering the transverse foramina at C3-C4 level.

\subsubsection{Anomalous branching}

This anomaly was found in 5 cases, with 4 cases originating from V3 rather than V4 PICA, while only 1 case was reported as bilateral and in 1 case Right AICA originating from V4 rather than basilar.

\subsubsection{Duplication}

Duplication was reported in only 1 male patient with 2 branches originating from the aortic arch and left Subclavian common Artery (SCA). Left VA was duplicated and conjoined at V2.

\subsubsection{Significant Stenosis}

In the present study, significant stenosis was reported in 23 cases (18.4\%). Origin of left VA was reported in 12 cases and origin of Rt VA was reported in 9 cases. In 2 cases, stenosis appeared at the distal of Lt VA, V4 segment of both Right and left VA before PICA (Table 3). 
Table 3

Significant Stenosis of VA

\begin{tabular}{|llllll|}
\hline Age group Sex & No Stenosis & Origin of Lt VA & Origin of Rt VA & Other & Total \\
\hline$<30$ Male & 2 & 0 & 0 & 0 & 2 \\
Female & - & 0 & 0 & 0 & 0 \\
\hline $30-40$ Male & 5 & 0 & 0 & 0 & 5 \\
Female & 9 & 0 & 0 & 0 & 9 \\
$40-60$ Male & 12 & 1 & 2 & 0 & 12 \\
Female & 23 & 0 & 0 & 1 & 24 \\
$60-80$ Male & 20 & 7 & 5 & 0 & 32 \\
Female & 27 & 2 & 2 & 0 & 31 \\
$>80$ Male & 2 & 1 & 0 & 1 & 4 \\
Female & 2 & 1 & 0 & 0 & 3 \\
\hline
\end{tabular}

\subsubsection{V4 Atresia, Thrombosis, Dissection}

In this study, 3 cases were reported as V4 Atresia in which all of them are on the right. Thrombosis was reported in 4 cases and dissection appeared in 4 cases of which 1 was reported to be bilateral.

\subsubsection{Hypoplasia}

In this study, hypoplasia was detected in 22 patients (17.6\%). hypoplasia found in 3 cases of V1-V3, 9 cases hypoplasia of V1-V4, 9 cases hypoplasia of V4 after PICA and 1 case hypoplasia of V4 (Table 4). 
Table 4

Hypoplasia of VA

\begin{tabular}{|lllllll|}
\hline Age group Sex & No Hypoplasia & V1-V3 & V1-V4 & V4 after PICA & V4 & Total \\
\hline$<30$ Male & 2 & 0 & 0 & 0 & 0 & 2 \\
Female & 0 & 0 & 0 & 0 & 0 & 0 \\
\hline $30-40$ Male & 5 & 0 & 0 & 0 & 0 & 5 \\
Female & 8 & 0 & 0 & 0 & 0 & 9 \\
\hline $40-60$ Male & 12 & 1 & 2 & 0 & 0 & 15 \\
Female & 20 & 0 & 1 & 2 & 1 & 24 \\
\hline $60-80$ Male & 26 & 1 & 0 & 5 & 0 & 32 \\
Female & 27 & 0 & 3 & 1 & 0 & 31 \\
\hline$>80$ Male & 1 & 1 & 2 & 0 & 0 & 4 \\
Female & 2 & 0 & 0 & 1 & 0 & 3 \\
\hline
\end{tabular}

In the case of VA dominance, $66.4 \%$ cases were co-dominant, while $16.0 \%$ were right, and $12.8 \%$ were left dominant. Most cases with a right or left dominant vertebral artery appeared in the 61-80 years age group (Table 1). Among patients, $4.0 \%(n=5)$ had an anomalous origin, of which 4 with left VA originated from the aortic arch, and in one patient, right VA originated from the right common carotid artery. Overall, males had more VA anomalies than females, $24.8 \%$ and $17.6 \%$, respectively (in general, $42.4 \%$ of all participants) ( $p=0.020)$. Referring to the anomalous route, in only one male patient did both VA enter the transverse foramina at the C3-C4 level. Besides, anomalous branching was reported in 5 patients (4.0\%), of whom four with posterior inferior cerebellar artery (PICA) originated from the $\mathrm{V} 3$ segment rather than the V4 segment. One patient with right anterior inferior cerebellar artery (AICA) derived from the V4 segment rather than the basilar artery. Duplication was seen in one male patient whose left VA was duplicated with two branches originating from the aortic arch and left subclavian artery, conjoint at the V2 segment.

The most common anomaly in the study population was significant stenosis, which was observed in 23 patients (18.4\%) at the origin of the left VA (12 patients), the origin of right VA (9 patients), distal left VA (1 patient), and V4 segments of both right and left VA before PICA. V4 atresia was also evident in three patients, all of whom were on the right side. VA hypoplasia was recorded in 22 patients (17.6\%) as follows: V1-distal V2, 3 cases; V1-distal V3, 9 cases; and V4. Dissection was found in four patients of which three were in the 41-60 years age group.

\section{Discussion}


Our findings revealed that approximately $42 \%$ of patients had at least one VA variation with a male preponderance. Co-dominance was the most commonly observed dominance. Among the variations, hypoplasia and significant stenosis were the most prevalent anomalies.

With advances in interventional radiology and surgical methods in the neck, a thorough knowledge of VA's anatomical variations regarding its origin, course, and branches is crucial to minimize procedurerelated complications [13]. Although colour Doppler ultrasonography has been traditionally considered the primary diagnostic modality in VA variations, CTA and MR angiography more accurately depict these variations and anomalies [14]. Coexisting intracerebral abnormalities in patients with VA variations should be investigated and vice versa, particularly in patients who experience cerebrovascular accidents [14].

Regarding dominance, the right outnumbered the left in our study. Our findings echoed those documented in previous studies, in which right and left dominance were observed in $45 \%$ and $30 \%$, respectively [1]. Some studies hypothesize that the VA's origin and diameter differences may result in cerebral disorders because of the altered cerebral haemodynamic [15]. Originating from the left aortic arch was observed in $3.2 \%$ of participants in this study. A left VA of aortic origin is correlated with a predilection for dissection, presumably due to the vessel's longer course in the neck [16]. This could also be attributed to congenital defects in the structural arterial wall and cerebral haemodynamic changes [14]. Our findings are consistent with previous studies that also reported a greater incidence of VA variation in males [16].

Significant origin stenosis was detected in approximately $18 \%$ of our patients. A stenotic VA origin is principally associated with arterial thromboembolism and hypoperfusion. These patients generally have coexisting atherosclerotic stenosis in both carotid and renal arteries [17]. A stenotic VA origin with the vessel's tortuousness of origin raises the probability of stent fracture during the placement procedure [18]. Posterior circulation stroke in about $5 \%$ of patients was linked to VA origin stenosis [19].

Regarding VA branching variations, PICA originated from V3 rather than V4 in $3.2 \%$ of our patients. After the VA enters the suboccipital region, meningeal branches emerge, and its terminal and largest branch the PICA - is given off [2]. Extradural origins of VA, similar to our findings, were reported in about $4 \%$ of patients through previous studies [20]. PICA termination is widespread in cases of left VA origin from the aortic arch, and the extradural branches of VA should be taken into consideration [13].

The present study reveals that the VA variations, especially significant stenosis ( 23 cases) and hypoplasia (22 cases), are the two most common variations with significant stenosis occurring more than hypoplasia. It is important to differentiate between acquired narrowing and hypoplasia of the VA because each of these has a different atherosclerotic burden [7].

\section{Conclusion}

In conclusion, our study shows that stenosis is more frequent than hypoplasia, so the former could be the most acquired and preventable factor of stroke. Besides, anatomical variations, including origin and 
branching, should be seriously considered in surgical or interventional radiology procedures to minimize the complications.

\section{Abbreviations}

vertebral artery (VA)

computed tomography angiography, CT-angiography (CTA)

anterior inferior cerebellar artery (AICA)

posterior inferior cerebellar artery (PICA)

Subclavian common Artery (SCA)

standard deviation (SD)

confidence interval $(\mathrm{Cl})$

posterior cerebellar stroke (PCS)

\section{Declarations}

\section{Ethics approval and consent to participate}

Ethical approval for this Study and use of patients three-dimensional computed tomography angiography (CTA) images for collection and study was obtained originally from the local ethics committee in Shahid Beheshti university of medical sciences, Tehran, Iran. The Iran National Committee for Ethics in Biomedical Research approved continuing this study.

We conducted this study using data and CTA images from Tooska Medical Imaging center. All study participants provided written informed consent for studying their images. Patients explained regarding how their clinical data would be used in the study process. The patients' information remained confidential and identities were anonymous.

\section{Consent for publication}

All images were performed in accordance with the relevant guidelines and regulations and written informed consent for studying and publication was obtained from all patients.

\section{Availability of data and material}

The computed tomography angiography (CTA) raw data generated and analyzed during the current study are available in the Dr Sofia Saburi repository, Radiologist and main director of Tooska imaging center, Email: dr.sabouri@hotmail.com. 


\section{Competing interests}

The authors declare that they have no known competing financial interests or personal relationships that could have appeared to influence the work reported in this paper.

\section{Funding}

None

\section{Authors' contributions}

This work was able to be completed due to the substantial contribution of the authors. All authors have contributed to the preparation and writing of the paper. All authors have read and approved the manuscript. F.M.1: Conceptualization, Writing-Original draft preparation, Writing- Reviewing and Editing, H.S: design of the work, supervising and editing, F.M.2: Methodology, interpretation of data, S.S: Reviewing and Editing, data availability.

Authors confirm that the manuscript has been read and approved by all named authors and that there are no other persons who satisfied the authorship criteria. Authors further confirm their unanimous approval of the order of the authors listed in the manuscript. Additionally, the authors declare that this manuscript is original, has not been published before, and is not currently being considered for publication elsewhere.

\section{Acknowledgements}

Not Applicable

\section{References}

1. C.W. Peng, B.T. Chou, J.A. Bendo, J.M. Spivak, Vertebral artery injury in cervical spine surgery: anatomical considerations, management, and preventive measures, The Spine Journal 9(1) (2009) $70-76$

2. T.H. Abd El-Bary, M. Dujovny, J.I. Ausman, Microsurgical anatomy of the atlantal part of the vertebral artery, Surgical neurology 44(4) (1995) 392-401

3. D.H. Padget, The development of the cranial arteries in the human embryo, Contrib Embryol 32 (1948) 205-261

4. R.A. Eisenberg, F.S. Vines, S.B. Taylor, Bifid origin of the left vertebral artery, Radiology 159(2) (1986) $429-430$

5. T.J. Kaufmann, J. Huston III, J.N. Mandrekar, C.D. Schleck, K.R. Thielen, D.F. Kallmes, Complications of diagnostic cerebral angiography: evaluation of 19826 consecutive patients, Radiology 243(3) (2007) 812-819

6. T.E. Nogueira, A.A. Chambers, M.T. Brueggemeyer, T.J. Miller, Dual origin of the vertebral artery mimicking dissection, American journal of neuroradiology 18(2) (1997) 382-384 
7. C. Kim, J.H. Sohn, H.C. Choi. Are the anomalous vertebral arteries more hypoplastic? retrospective linear mixed model approach. BMC Neurol Journal. 2017 Aug 29;17(1):168.

8. Y.M. Chuang, Y.C. Huang, H.H. Hu, et al. Toward a further elucidation: role of vertebral artery hypoplasia in acute ischemic stroke. Eur Neurol. 2005;55: 193-7.

9. M. Arnold, M.G. Bousser, G. Fahrni, U. Fischer, D. Georgiadis, J. Gandjour, D. Benninger, M. Sturzenegger, H.P. Mattle, R.W. Baumgartner, Vertebral artery dissection: presenting findings and predictors of outcome, Stroke 37(10) (2006) 2499-2503

10. W.I. Schievink, Spontaneous dissection of the carotid and vertebral arteries, New England Journal of Medicine 344(12) (2001) 898-906

11. R.W. Molinari, P.C. Chimenti, R. Molinari Jr, W. Gruhn, Vertebral artery injury during routine posterior cervical exposure: case reports and review of literature, Global spine journal 5(6) (2015) 528-532

12. A. Stephanie, DeCarvalho, M. Muhammad, Abd-El-Barr, Michael W.Groff. Vascular Complications in Cervical Spine Surgery (Anterior and Posterior Approach). Complications in Neurosurgery, 2019

13. E.-P. Magklara, E.-T. Pantelia, E. Solia, E. Panagouli, M. Piagkou, A. Mazarakis, P. Skandalakis, T. Troupis, D. Filippou, Vertebral artery variations revised: origin, course, branches and embryonic development, Folia Morphologica (2020)

14. N. Lazaridis, M. Piagkou, M. Loukas, E.-T. Piperaki, T. Totlis, G. Noussios, K. Natsis, A systematic classification of the vertebral artery variable origin: clinical and surgical implications, Surgical and Radiologic Anatomy 40(7) (2018) 779-797

15. L. Bernardi, P. Dettori, Angiographic study of a rare anomalous origin of the vertebral artery, Neuroradiology 9(1) (1975) 43-47

16. J. Dumfarth, A.S. Chou, B.A. Ziganshin, R. Bhandari, S. Peterss, M. Tranquilli, H. Mojibian, H. Fang, J.A. Rizzo, J.A. Elefteriades, Atypical aortic arch branching variants: a novel marker for thoracic aortic disease, The journal of thoracic and cardiovascular surgery 149(6) (2015) 1586-1592

17. R. Mohammadian, A. Najaran, B. Sohrabi, R. Mansourizadeh, F. Mohammadian, B. Nasiri, M. Farhoudi, Vertebral artery orifice stenosis: a report of 43 cases from northwest iran treated with angioplasty and stenting, The Neuroradiology Journal 24(5) (2011) 749-757

18. S.-R. Kim, M.-W. Baik, S.-H. Yoo, I.-S. Park, S.-D. Kim, M.-C. Kim, Stent fracture and restenosis after placement of a drug-eluting device in the vertebral artery origin and treatment with the stent-in-stent technique: report of two cases, Journal of neurosurgery 106(5) (2007) 907-911

19. G.L.G. Perue, R. Narayan, A.H. Zangiabadi, J.G. Romano, T. Rundek, R.L. Sacco, S. Koch, Prevalence of vertebral artery origin stenosis in a multirace-ethnic posterior circulation stroke cohort: Miami Stroke Registry (MIAMISR), International Journal of Stroke 10(2) (2015) 185-187

20. H. Krayenbühl, M.G. Yaşargil, Die vaskulären Erkrankungen im Gebiet der arteria vertebralis und arteria basialis: eine anatomische und pathologische, klinische und neuroradiologische Studie: mit 127 Abbildungen in 205 Einzeldarstellungen, G. Thieme1957. 